\title{
DINÂMICA DA REGENERAÇÃO NATURAL EM TRÊS ESTÁGIOS SUCESSIONAIS DE UMA FLORESTA OMBRÓFILA DENSA EM BLUMENAU, SC
}

\author{
Lauri Amândio Schorn*, Franklin Galvão** \\ *Eng. Florestal, Dr., Depto. de Engenharia Florestal, FURB - 1schorn@furb.br \\ **Eng. Florestal, Dr., Depto. de Ciências Florestais, UFPR, Pesquisador do CNPq - fgalvão@ufpr.br \\ Recebido para publicação: 11/11/2005 - Aceito para publicação: 25/04/2006
}

\begin{abstract}
Resumo
Este trabalho foi realizado com o objetivo de avaliar a dinâmica da regeneração natural de indivíduos arbóreos e arborescentes em Floresta Ombrófila Densa Submontana, nos estágios sucessionais inicial, intermediário e avançado, no Parque Natural Nascentes do Garcia, no município de Blumenau, SC. Para isso, foram instaladas, em 2001, 20 unidades amostrais permanentes de $10 \times 3 \mathrm{~m}$ em cada estágio sucessional, com o propósito de avaliar os indivíduos com circunferência à altura do peito inferior a $15 \mathrm{~cm}$ e altura superior a $50 \mathrm{~cm}$. Circunscritas a essas unidades e em igual número, sub-unidades permanentes de $10 \times 1 \mathrm{~m}$ foram alocadas para avaliar indivíduos com altura entre 10 e $50 \mathrm{~cm}$. Todos os indivíduos amostrados foram identificados e tiveram suas alturas medidas. Em 2003, usando os mesmos critérios de inclusão, foi realizada uma nova mensuração, o que permitiu avaliar mortalidade e ingresso. Como resposta, verificou-se que a mortalidade, o ingresso, bem como a relação ingresso/mortalidade são maiores nos estágios iniciais e diminuem gradualmente com o desenvolvimento da vegetação. Entre os grupos ecológicos, de forma geral, as espécies pioneiras foram as que apresentaram maiores taxas de mortalidade em relação aos ingressos. As espécies clímax tolerantes à sombra mostraram um dinamismo progressivo do estágio inicial para o avançado. No estágio inicial, os maiores ingressos foram de espécies clímax exigentes em luz, ao passo que nos estágios intermediário e avançado as espécies clímax tolerantes à sombra apresentaram os maiores ingressos.
\end{abstract}

Palavras-chave: Regeneração; mortalidade; ingresso; sucessão.

\section{Abstract}

Dynamics of natural regeneration in three stages of an Atlantic Rain Forest in Blumenau, SC. This work, which was developed in the Natural Park called Nascentes do Garcia in Blumenau city, Santa Catarina, had the objective to evaluate the dynamic of the Submontain Atlantic Rain Forest, in the initial, intermediate and late successional stages. To do that, in 2001, 20 permanent sample plots of 10 x $3 \mathrm{~m}$ size were installed in each successional stage to evaluate the individuals which contained a breast height circumference of $15 \mathrm{~cm}$ size and a height superior to $50 \mathrm{~cm}$. Inside this sample plots, 20 permanent sample plots of $10 \times 1 \mathrm{~m}$ size were installed to evaluate individuals between 10 and $50 \mathrm{~cm}$ height. All the individuals were identified and had their height measured. In 2003, using the same inclusion criteria, the sample plots were re-measured, allowing to evaluate the mortality and ingrowth changes. The results indicated that the mortality, the ingrow, and the relation ingrow/mortality were higher in the initial stages and gradually decreased with the vegetation development. Among the ecologic groups, in general, the pioneer species showed the highest mortality taxes in comparison to the ingrow. The shadow tolerant climax species showed a progressive dynamism from the initial stage to the late one. In the initial stage, the light demanding climax species had more expressive ingrow, and in the intermediate and late stages, the shade tolerant climax species showed the highest ingrowth.

Keywords: Regeneration; mortality; ingrow; succession. 


\section{INTRODUÇÃO}

O compartimento arbóreo inferior da floresta apresenta-se, em geral, com um comportamento diferenciado em muitos aspectos da estrutura e da dinâmica. Pode-se destacar, entre esses aspectos, a densidade, as taxas de ingresso e mortalidade, além do crescimento.

A expressão "regeneração natural" tem um conceito de avaliação muito amplo. Para Finol (1971), todos os descendentes de plantas arbóreas que se encontram entre 0,10 $\mathrm{m}$ de altura até o limite de $10 \mathrm{~cm}$ de diâmetro à altura do peito (DAP) são definidos como indivíduos de regeneração natural. Rollet (1978) e Volpato (1994) consideram como regeneração natural todos os indivíduos com DAP inferior a 5 cm. Rollet (1978) considera ainda como regeneração as fases juvenis das espécies, em que cada classe diamétrica se constitui em regeneração da fração da população da mesma espécie com diâmetro superior a essa classe.

O potencial de regeneração das espécies em um determinado fragmento florestal (resiliência) é variável no espaço e no tempo, podendo promover, quando eficientemente manejado, a restauração parcial ou total da vegetação na área remanescente (RODRIGUES, 1999). Dentro desse contexto, a heterogeneidade da resiliência determina a composição do mosaico vegetacional de um remanescente florestal, no qual cada unidade vai ser ocupada por diferentes conjuntos de espécies, dependendo das características dessa unidade em termos de tamanho, época de abertura e contexto no qual está inserida, entre outros determinantes (PICKETT; OSTFELD, 1995).

Entre os fatores que controlam o desenvolvimento da regeneração natural em populações de florestas tropicais, Whitmore $(1975)^{1}$, citado por Primack e Lee (1991), refere-se à luz que atinge os estratos inferiores da floresta, citando ainda que, sendo o ambiente de luz extremamente heterogêneo, conduz a respostas diferentes das espécies, pois cada uma responde otimamente a determinada intensidade de luz.

A intensidade de luz próxima à superfície do solo, que sofre abrupta elevação quando árvores ou galhos caem, formando uma clareira, retorna novamente a níveis baixos no período de 5 a 10 anos. A abertura de clareiras e seus processos associados ocasionam flutuações na luz, que influenciam no crescimento dos indivíduos da regeneração. Estes respondem às flutuações de luz através de mudanças no crescimento, arquitetura e fisiologia (STERCK et al., 1999).

Richard (1952) e Brokaw (1985a) afirmam que espécies de floresta primária são tolerantes a sombra somente de forma limitada; elas dependem do surgimento de clareiras para chegar à maturidade, pois, no sub-bosque, a mortalidade de plântulas é alta, e somente algumas plântulas sobrevivem.

As espécies pioneiras na fase juvenil são distinguidas das facultativas ou climácicas quando são encontradas somente em clareiras de uma certa magnitude. Dessa forma, clareiras são tipicamente colonizadas por pioneiras. Entre as pioneiras, são distinguidas duas classes: de ciclo curto e de ciclo longo. A classe de ciclo curto, com comportamento pioneiro mais típico, invade mais rapidamente grandes clareiras naturais e áreas abertas; já as pioneiras de ciclo longo (secundárias) têm menores distinções em relação às espécies facultativas, pois também ocupam clareiras de pequeno tamanho (BROKAW, 1985a).

Grubb (1977) menciona o termo "nicho de regeneração", como uma expressão das necessidades de uma espécie para obter uma elevada probabilidade de sucesso na substituição de um indivíduo maduro por um novo indivíduo da nova geração. De acordo com esse conceito, em muitas comunidades as diferenças de nicho entre espécies coexistentes podem se manifestar somente durante os primeiros estágios de desenvolvimento, o que significa que as necessidades para os processos como a iniciação da reprodução vegetativa, dispersão de propágulos, germinação, estabelecimento de plântulas e crescimento de indivíduos jovens podem diferir para espécies que aparentemente têm necessidades semelhantes quando os indivíduos são maduros.

A dinâmica da regeneração natural vai depender da extensão e do tipo de perturbação, da proximidade onde se encontram as fontes produtoras de propágulos, da disponibilidade de agentes dispersores, da herbivoria de plântulas, da competição com gramíneas agressoras (TORIOLA et al.,

\footnotetext{
${ }^{1}$ Whitmore, T. C. Macaranga Pp 140-159 in Airy-Shaw, H. K. (ed.) The Euphorbiaceae of Borneo. Kew Bulletin Additional Séries IV. Royal Botanical Gardens, Kew.1975.
} 
1998), das condições microclimáticas, dos aspectos físicos e químicos do solo e da compactação deste (PARROTA, 1993²; HOLL; KAPPELLE, $1999^{3}$, citados por SORREANO, 2002).

Carvalho (1997) comenta que alguns estudos apontam taxas de mortalidade mais baixas em espécies emergentes, enquanto que em espécies de sub-bosque essas taxas são mais altas. Além disso, outros estudos considerando apenas os indivíduos com DAP $\geq 10 \mathrm{~cm}$ não indicam nenhuma diferença em mortalidade por classes de tamanho.

A mortalidade mais intensa em plantas ocorre no estágio juvenil, conforme mencionaram Harper (1977) e Cook (1979) ${ }^{4}$, citado por Still (1996). Still (1996) complementa que, em florestas, os processos que formam a futura composição de espécies atuam mais fortemente sobre as plântulas.

Whitmore (1984, 1990), Brokaw (1985a) e Denslow (1987) consideram que o crescimento de plântulas sobreviventes depende do aumento de intensidade de luz, resultante da abertura do dossel, e espécies arbóreas diferem no total de luz necessária para incrementar o crescimento.

Relatando o efeito de uma clareira no microambiente, Popma e Bongers (1991) afirmaram que ele é mais pronunciado na regeneração de espécies tolerantes à sombra de uma floresta. Complementaram afirmando que as diferenças em crescimento entre espécies, como respostas ao estímulo de clareiras, são mais pronunciadas durante os primeiros estágios do ciclo de vida (germinação, estabelecimento de plântulas e crescimento).

$\mathrm{Na}$ ausência de distúrbios em larga escala, a dinâmica da regeneração é fortemente influenciada por fatores endógenos (estrutura da vegetação e interação entre espécies), que operam em escalas de milhares de metros quadrados ou menores. Fatores controlando a dinâmica da regeneração nessa escala incluem: clareiras causadas por quedas de árvores; mortalidade de sementes em germinação e de plântulas, causada por patógenos, herbívoros e predadores de sementes; curta distância de dispersão, alelopatia e heterogeneidade local no ambiente físico (AUGSPURGER, 1984; VEBLEN, 1992).

As espécies diferem nas respostas, não somente quanto à abertura do dossel, mas também em relação à suscetibilidade a patógenos, herbívoros e predadores. Essas variações no comportamento das espécies reduzem a exclusão competitiva ao nível de comunidades e têm sido consideradas como fatores que contribuem para a manutenção da biodiversidade em riqueza de espécies florestais (DENSLOW, 1987; HUSTON, 19945 , e JANZEN ${ }^{6}$, 1970, citados por GRAU, 2000).

A distância de dispersão de sementes a partir da árvore-matriz, particularmente em espécies com dispersão anemófila, decresce de acordo com uma curva exponencial negativa. Isso implica que a chuva de sementes é extremamente agregada em poucos metros ao redor de árvores adultas. Conseqüentemente, a expectativa de mortalidade causada por patógenos, herbívoros e predadores é muito maior nas proximidades de árvores maduras (WILSON, 19937 JANZEN, 1970 ${ }^{8}$, citados por GRAU, 2000). No entanto, Veblen (1992) menciona que podem ocorrer padrões diversos de distribuição de plântulas em função da dispersão de sementes e relata o modelo proposto por Hubbell $(1980)^{9}$, que sugere um recrutamento mais elevado próximo à planta-matriz, onde a densidade de sementes é maior, embora poucas sobrevivam.

O conhecimento da composição da regeneração contribui para definir o estágio, bem como as direções sucessionais de uma vegetação em desenvolvimento. A avaliação da dinâmica da regeneração, através dos ingressos, mortalidade e crescimento, fornece informações que permitem identificar as espécies que deverão ter maior importância no futuro, bem como aquelas que tendem a diminuir a participação na estrutura.

\footnotetext{
${ }^{2}$ Parrota, J. A. Secondary Forest regeneration on degraded tropical lands: the role of plantations as "foster ecosystems". In: Lieth, H.; Lohmann, M. (Ed.) Restoration of tropical forest ecosystems. The Haque: Kluwer Academic, 1993

${ }^{3}$ Holl, K. D.; Kappelle, M. Tropical forest recovery and restoration. Trends in Ecology and Evolution. V. 14, n. 10, p. 378-379, 1999.

${ }^{4}$ Cook, R. E. Patterns of juvenile mortality and recruitment in plants. In Solbrig, O. T.; Jain, S.; Johnson, G. B.; Raven, P. H.

(Eds.), Topics in Plant Population Biology, pp. 207-231. Columbia University Press, New York. 1979

${ }^{5}$ HUSTON, M. Biological Diversity. The coexistence of species in changing landscapes. Cambridge University Press, Cambridge, UK, 1994. $430 \mathrm{p}$.

${ }^{6}$ JANZEN, D.H. Herbivores and the number of tree species in tropical forests. American Naturalists. 104:501-527. 1970.

${ }^{7}$ WILSON, M.F. Dispersal mode, seed shadows and colonization patterns. Vegetatio 107/108:261-280.

${ }^{8}$ Op. Cit.

${ }^{9}$ HUBBELL, S.P. Seed predation and the coexistence of tree species in tropical forests. Oikos 35:214-229. 1980.
} 
O conhecimento das direções sucessionais de comunidades vegetais oferece importantes subsídios para o planejamento da recuperação de áreas degradadas, bem como para o conhecimento da resiliência de florestas.

Neste trabalho, o objetivo foi avaliar a dinâmica da regeneração natural das espécies em três fases sucessionais de uma Floresta Ombrófila Densa Submontana, através da análise da mortalidade e ingressos, além dos incrementos em altura.

\section{MATERIAL E MÉTODOS}

\section{Localização}

A floresta, objeto do estudo, faz parte do Parque Natural Nascentes do Ribeirão Garcia, situado no município de Blumenau, Santa Catarina, e pertence à Universidade Regional de Blumenau e à Fundação Municipal do Meio Ambiente.

O Parque das Nascentes possui uma área de 5.300 ha, com 98\% de cobertura arbórea, caracterizada por diferentes estágios de sucessão da Floresta Ombrófila Densa. Distante cerca de $20 \mathrm{~km}$ do centro de Blumenau, está localizado entre as coordenadas $27^{\circ} 01^{\prime}$ e $27^{\circ} 06^{\prime}$ S e $49^{\circ} 01^{\prime}$ e $49^{\circ} 10^{\prime}$ W, em altitude de 350 a 650 m s.n.m (Figura 1).

\section{Obtenção dos dados}

A amostragem da vegetação foi feita em três estágios sucessionais - inicial, intermediário e avançado -, todos situados na sub-bacia hidrográfica do ribeirão Encano, no extremo norte do Parque. A vegetação dos estágios inicial e intermediário era secundária, enquanto que do avançado possivelmente primária alterada.

Para avaliar as árvores com CAP maior ou igual a $15 \mathrm{~cm}$, foram utilizadas 20 unidades amostrais retangulares de $10 \times 20 \mathrm{~m}$ em cada estágio sucessional, totalizando 60 unidades amostrais.

Foram demarcadas 20 unidades amostrais permanentes de 10 × $3 \mathrm{~m}$ em cada estágio sucessional, onde foram avaliados os indivíduos com circunferência à altura do peito (CAP) inferior a $15 \mathrm{~cm}$ e altura superior a $50 \mathrm{~cm}$. Circunscritas a essas unidades e em igual número, sub-unidades permanentes de $10 \mathrm{x} 1$ $\mathrm{m}$ foram alocadas para avaliar indivíduos com altura entre 10 e $50 \mathrm{~cm}$.

As dimensões das unidades e sub-unidades amostrais foram determinadas considerando-se resultados obtidos em outros levantamentos fitossociológicos realizados na Floresta Ombrófila Densa (VIBRANS, 1999; SALIMON; NEGRELLE, 2001; SCHORN; GALVÃO, 2002; SEVEGNANI, 2003).

A amostragem foi realizada em duas ocasiões: a primeira, entre dezembro de 2000 e maio de 2001; a segunda, entre dezembro de 2002 e maio de 2003.

Todos os indivíduos amostrados foram identificados com uma plaqueta enumerada afixada em um de seus galhos ou no caule, no caso de plantas menores, e tiveram suas alturas medidas com o auxílio de varas graduadas. Por ocasião da segunda amostragem, usando os mesmos critérios de inclusão, foram mensuradas as alturas dos indivíduos vivos, sendo incluídos e mensurados aqueles que atingiram a altura de $10 \mathrm{~cm}$ após a primeira avaliação. Os indivíduos mensurados na primeira ocasião e não presentes na segunda ocasião foram considerados mortos.

Foi coletado material botânico fértil ou não para herborização e posterior identificação das espécies encontradas. Esse material foi submetido e registrado nos herbários dos cursos de Engenharia Florestal da Universidade Regional de Blumenau (Departamento de Engenharia Florestal) e da Universidade Federal do Paraná (Departamento de Ciências Florestais).

Com os dados coletados em campo, foram efetuados os cálculos de densidade por hectare e por espécie, bem como avaliadas as suas alterações nas duas ocasiões de levantamento, em cada estágio sucessional amostrado.

A dinâmica da regeneração natural foi analisada para cada espécie e para o total, através dos parâmetros de ingresso e da mortalidade de indivíduos. As médias de ingressos e mortalidade, por grupo ecológico e nos três estágios sucessionais, foram comparadas através do teste $\mathrm{F}$, ao nível de $95 \%$ de significância. 


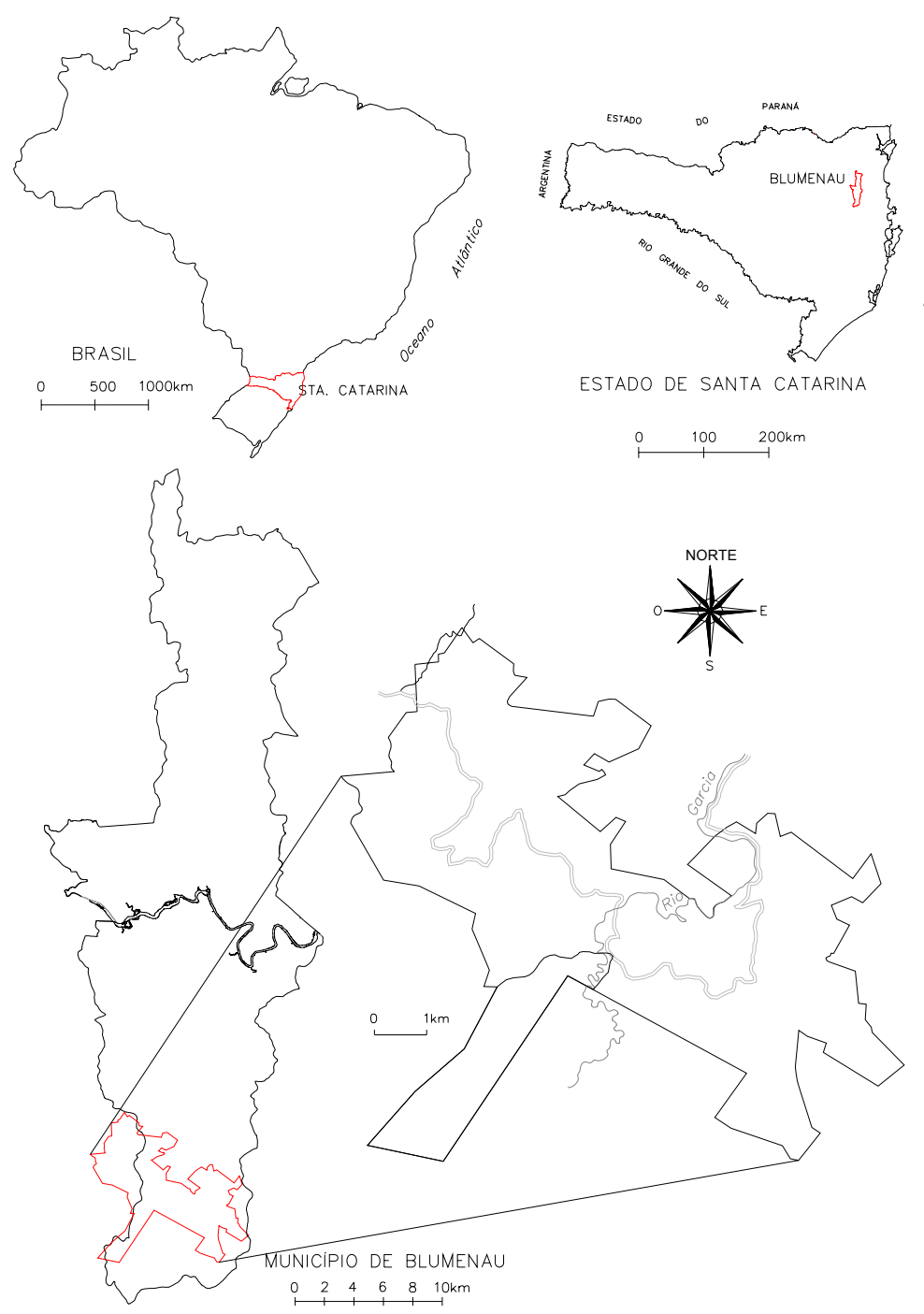

Figura 1. Localização da área de estudos.

Figure 1. Localization of the study area.

\section{RESULTADOS E DISCUSSÕES}

Os ingressos e as mortalidades por espécie estão representados nas tabelas 1,2 e 3, respectivamente para os estágios inicial, intermediário e avançado.

\section{Estágio inicial}

No estágio inicial, os maiores ingressos relativos e absolutos foram de Miconia cabucu, Miconia cinnamomifolia e Hieronyma alchorneoides, seguidas ainda de Piper gaudichaudianum, Myrcia pubipetala e Alchornea glandulosa (Tabela 1). No período de dois anos, houve um aumento de 3975 novos índivíduos por hectare, o que representa um aumento de 38,97\% em relação ao total das espécies para essa fase sucessional. Os menores ingressos absolutos foram observados em Matayba guianensis e Cytharexyllum myrianthum (25 ind./ha) e em Vernonia sp. (50 ind./ha). Já os menores ingressos relativos foram encontrados para Matayba guianensis (2\%), Cytharexyllum myrianthum (5\%) e Xylopia brasiliensis (14\%). 
Tabela 1. Ingressos, mortalidade e porcentagem de mudanças, por espécie, no estágio inicial.

Table 1. Ingrow, mortality and percent of changes, by species in the initial stage.

\begin{tabular}{|c|c|c|c|c|c|c|c|c|}
\hline \multirow{2}{*}{ Espécies } & \multicolumn{2}{|c|}{ Densidade } & \multicolumn{2}{|c|}{ Ingressos } & \multicolumn{2}{|c|}{ Mortalidade } & \multirow{2}{*}{$\begin{array}{c}\text { Mudança } \\
\%\end{array}$} & \multirow{2}{*}{$\begin{array}{c}\text { Grupo } \\
\text { ecológico }\end{array}$} \\
\hline & 2001 & 2003 & n.hat ${ }^{-1}$ & $\%$ & n.ha ${ }^{-1}$ & $\%$ & & \\
\hline Alchornea glandulosa & 275 & 450 & 175 & 64 & & & 63,6 & $\mathrm{CL}$ \\
\hline Alchornea triplinervia & 100 & 150 & 75 & 75 & 25 & 25 & 50,0 & $\mathrm{CL}$ \\
\hline Annona cacans & 25 & 25 & & & & & & $\mathrm{CL}$ \\
\hline Campomanesia sp. & 150 & 275 & 125 & 83 & & & 83,3 & \\
\hline Casearia decandra & 225 & 350 & 125 & 56 & & & 55,6 & $\mathrm{P}$ \\
\hline Cecropia glaziovii & 25 & 25 & & & & & & $\mathrm{P}$ \\
\hline Clusia criuva & 50 & 50 & & & & & & $\mathrm{P}$ \\
\hline Cytharexyllum myrianthum & 175 & 200 & 25 & 14 & & & 14,3 & $\mathrm{P}$ \\
\hline Dodonea viscosa & 150 & 125 & 25 & 17 & 50 & 33 & $-16,7$ & $P$ \\
\hline Hieronyma alchorneoides & 1025 & 1475 & 450 & 44 & & & 43,9 & $\mathrm{CL}$ \\
\hline Matayba guianensis & 200 & 225 & 25 & 13 & & & 12,5 & CL \\
\hline Miconia cabucu & 525 & 1600 & 1075 & 205 & & & 204,8 & $\mathrm{CL}$ \\
\hline Miconia cinnamomifolia & 1250 & 1675 & 725 & 58 & 300 & 24 & 34,0 & $\mathrm{P}$ \\
\hline Myrcia pubipetala & 400 & 575 & 175 & 44 & & & 43,8 & $\mathrm{CS}$ \\
\hline Myrsine coriacea & 1475 & 1050 & 75 & 5 & 500 & 34 & $-28,8$ & $\mathrm{P}$ \\
\hline Ocotea puberula & 75 & 75 & & & & & & $\mathrm{P}$ \\
\hline Ocotea teleiandra & 25 & 25 & & & & & & $\mathrm{CL}$ \\
\hline Ormosia arborea & 50 & 50 & & & & & & $\mathrm{CL}$ \\
\hline Piper gaudichaudianum & 2300 & 1900 & 275 & 12 & 675 & 29 & $-17,4$ & $\mathrm{CS}$ \\
\hline Psidium guajava & 225 & 225 & & & & & & $\mathrm{P}$ \\
\hline Psychotria nuda & & 100 & 100 & & & & & $\mathrm{CS}$ \\
\hline Sorocea bonplandii & 50 & 100 & 50 & 100 & & & 100,0 & $\mathrm{CS}$ \\
\hline Talauma ovata & 25 & 125 & 100 & 400 & & & 400,0 & $\mathrm{CL}$ \\
\hline Tibouchina pulchra & 250 & 275 & 100 & 40 & 75 & 30 & 10,0 & $\mathrm{P}$ \\
\hline Trema micrantha & 25 & 25 & & & & & & $\mathrm{P}$ \\
\hline Vernonia puberula & 375 & 425 & 125 & 33 & 75 & 20 & 13,3 & $\mathrm{P}$ \\
\hline Vernonia sp. & 575 & 500 & 50 & 9 & 125 & 22 & $-13,0$ & $P$ \\
\hline Virola bicuhyba & 25 & 25 & & & & & & $\mathrm{CL}$ \\
\hline Xylopia brasiliensis & 150 & 250 & 100 & 67 & & & 66,7 & $\mathrm{CL}$ \\
\hline Total & 10200 & 12350 & 3975 & & 1825 & & 15,7 & \\
\hline
\end{tabular}

$\mathrm{P}=$ pioneiras; $\mathrm{CL}=$ clímax exigentes em luz; $\mathrm{CS}=$ clímax tolerantes à sombra.

A mortalidade nesse estágio sucessional foi de 1825 ind./ha, representada principalmente por Piper gaudichaudianum, Myrsine coriacea, Miconia cinnamomifolia e Vernonia sp., que apresentaram mortalidade superior a 100 ind./ha no período. Os maiores valores relativos de mortalidade foram encontrados para Myrsine coriacea, Dodonea viscosa e Tibouchina pulchra, todas com mortalidade igual ou superior a $30 \%$.

De forma geral, as espécies pioneiras, amplamente dominantes nessa fase, não manifestaram os maiores valores em ingressos. Esses foram obtidos pelas espécies clímax exigentes em luz, representadas especialmente por Miconia cabucu e Hieronyma alchorneoides.

Por outro lado, a mortalidade foi representada principalmente pelas pioneiras, especialmente pelas espécies Myrsine coriacea, Miconia cinnamomifolia e Vernonia sp. Esses resultados demonstram que, no estrato da regeneração natural, as espécies pioneiras estão sendo gradualmente substituídas pelas clímax exigentes em luz e em menor escala pelas clímax tolerantes à sombra. Essa tendência também foi relatada por Brokaw (1985), que destacou a notável mortalidade de pioneiras quando a vegetação torna-se mais densa, que tem como causa principal a intensa competição pela luz entre espécies colonizadoras.

O fato acima também pode ser observado na porcentagem de mudança (Tabela 1), pela qual se verifica que, com exceção de Piper gaudichaudianum, as mudanças negativas de densidade foram manifestadas em espécies pioneiras. Por outro lado, as maiores mudanças positivas foram de Talauma 
ovata, Sorocea bonplandii, Miconia cabucu, Campomanesia sp., Xylopia brasiliensis, Alchornea glandulosa e Casearia decandra, notadamente espécies exigentes em luz e tolerantes à sombra.

\section{Estágio intermediário}

Nessa fase sucessional, ingressaram 3.800 ind./ha no período de dois anos, que correspondeu a um acréscimo de 5,18\% em relação à densidade em 2001 (Tabela 2).

As espécies que tiveram maiores ingressos no período foram Psychotria nuda, Psychotria longipes, Myrcia pubipetala, Matayba guianensis, Inga sp.1, Lonchocarpus campestris e Marlierea tomentosa, todas com ingressos iguais ou superiores a 200 indivíduos por hectare no período.

Os ingressos relativos, considerados em relação à densidade das espécies em 2001, foram mais expressivos em Ficus sp., Alchornea glandulosa, Machaerium stipitatum, Faramea marginata e Psychotria nuda, cujos valores foram iguais ou superiores a $25 \%$ de ingressos. Observa-se também que algumas espécies tiveram ingresso absoluto elevado, mas com ingresso relativo entre os mais baixos, como é ocaso de Myrcia pubipetala e Psychotria longipes. Por outro lado, Alchornea glandulosa, que teve o maior ingresso relativo, está entre as espécies com o menor ingresso absoluto.

Esses resultados mostram que as mudanças relativas na densidade são maiores nas espécies com populações pequenas, embora essas tenham, em geral, importância menor na estrutura da floresta ou do estrato analisado, razão pela qual esses resultados devem ser vistos com cautela.

A mortalidade nessa fase foi de 4.050 indivíduos por hectare, correspondendo a 5,52\% da população existente em 2001. Foi representada principalmente por Psychotria longipes, Myrcia pubipetala, Piper gaudichaudianum, Sloanea sp. e Inga sp.1, com valores iguais ou superiores a 250 indivíduos mortos por hectare.

A mortalidade relativa foi mais expressiva em Guatteria australis, Sapium glandulatum, Virola bicuhyba e Miconia cinnamomifolia, com valores iguais ou superiores a $24 \%$ no período. No estágio intermediário, tanto os ingressos quanto a mortalidade foram mais expressivos nas espécies clímax tolerantes à sombra, representando, respectivamente, $69 \%$ e $64 \%$ dos ingressos e mortalidades nessa fase (Figuras 2 e 3).

Esses resultados indicam que as espécies mais importantes no estrato superior da estrutura atual do estágio intermediário, notadamente pioneiras e clímax exigentes em luz, serão substituídas no futuro pelas espécies que atualmente apresentam maiores ingressos e maior relação ingressos/mortalidade na regeneração natural.

\section{Estágio avançado}

O ingresso no estágio avançado foi de 3.525 ind./ha, correspondendo a $14,90 \%$ em relação à densidade em 2001 (Tabela 3).

Os maiores ingressos na floresta primária alterada foram verificados em Euterpe edulis (1525), Myrcia pubipetala (525), Inga sp.1 (250), Mollinedia schottiana (200), Matayba guianensis (150) e Esenbeckia sp. (125). Outras 18 espécies apresentaram ingressos nessa floresta inferiores a 125 indivíduos por hectare no período.

Os ingressos foram menos expressivos na regeneração das espécies pioneiras e clímax exigentes em luz, com até $125 \mathrm{ind}$./ha/ano nesses grupos. Nas espécies clímax tolerantes à sombra, o número de ingressantes foi bastante superior, com 2.650 indivíduos por hectare (Tabela 3).

A mortalidade na floresta primária alterada foi de 1.650 ind./ha, representada principalmente pelas espécies Euterpe edulis (400) e Psychotria longipes (200). Em relação à população de 2001, os maiores valores relativos de mortalidade foram encontrados em Protium kleini, Mollinedia schottiana, Xylopia brasiliensis e Ormosia arborea, todas com 100\%. Entre as demais espécies, 18 outras também apresentaram mortalidade, mas com valores inferiores a $34 \%$ no período (Tabela 3 ).

$\mathrm{Na}$ distribuição da mortalidade nos grupos ecológicos, os resultados mostraram que ela foi expressivamente superior nas espécies climácicas tolerantes à sombra, em relação às pioneiras e climácicas exigentes em luz (Figura 3). Nessa fase, a estrutura da regeneração natural é amplamente dominada pelas espécies climácicas tolerantes à sombra, o que justifica, de certa forma, a ocorrência inexpressiva de ingressos e mortalidade em espécies pioneiras e em climácicas exigentes em luz. 
Tabela 2. Ingressos, mortalidade e porcentagem de mudanças, por espécie, no estágio intermediário.

Table 2. Ingrow, mortality and percent of changes, by species, in the intermediate stage.

\begin{tabular}{|c|c|c|c|c|c|c|c|c|}
\hline \multirow{2}{*}{ Espécies } & \multicolumn{2}{|c|}{ Densidade } & \multicolumn{2}{|c|}{ Ingressos } & \multicolumn{2}{|c|}{ Mortalidade } & \multirow{2}{*}{$\begin{array}{c}\text { Mudança } \\
\%\end{array}$} & \multirow{2}{*}{$\begin{array}{c}\text { Grupo } \\
\text { ecológico }\end{array}$} \\
\hline & 2001 & 2003 & n.ha ${ }^{-1}$ & $\%$ & n.hat ${ }^{-1}$ & $\%$ & & \\
\hline Aegiphylla sellowiana & 50 & 50 & & & & & & $\mathrm{P}$ \\
\hline Alchornea glandulosa & 75 & 125 & 50 & 67 & & & 67 & $\mathrm{CL}$ \\
\hline Alchornea triplinervia & 25 & 25 & & & & & & $\mathrm{CL}$ \\
\hline Allophylus edulis & 125 & 125 & & & & & & $\mathrm{CS}$ \\
\hline Annona cacans & 75 & 75 & & & & & & $\mathrm{CL}$ \\
\hline Aspidosperma camporum & 25 & 25 & & & & & & $\mathrm{P}$ \\
\hline Bathysa meridionalis & 300 & 275 & & & 25 & 8 & -8 & $\mathrm{CS}$ \\
\hline Cabralea canjerana & 125 & 125 & & & & & & $\mathrm{CS}$ \\
\hline Calyptranthes lucida & 50 & 50 & & & & & & $\mathrm{CS}$ \\
\hline Casearia decandra & 175 & 150 & & & 25 & 14 & -14 & $\mathrm{CL}$ \\
\hline Casearia sylvestris & 25 & 25 & & & & & & $\mathrm{CL}$ \\
\hline Cedrela fissilis & 50 & 50 & & & & & & $\mathrm{CL}$ \\
\hline Celtis sp. & 50 & 50 & & & & & & \\
\hline Clusia criuva & 550 & 575 & 50 & 9 & 25 & 5 & 5 & $P$ \\
\hline Endlicheria paniculata & 50 & 50 & & & & & & $\mathrm{CS}$ \\
\hline Esenbeckia grandiflora & 250 & 300 & 50 & 20 & & & 20 & $\mathrm{CS}$ \\
\hline Eugenia sp. & 50 & 50 & & & & & & $\mathrm{CS}$ \\
\hline Euterpe edulis & 825 & 925 & 150 & 18 & 50 & 6 & 12 & $\mathrm{CS}$ \\
\hline Faramea marginata & 375 & 475 & 100 & 27 & & & 27 & $\mathrm{CS}$ \\
\hline Ficus sp. & 25 & 75 & 50 & 200 & & & 200 & \\
\hline Geonoma gamiova & 75 & 75 & & & & & & $\mathrm{CS}$ \\
\hline Guapira opposita & 1875 & 1775 & & & 100 & 5 & -5 & $\mathrm{CS}$ \\
\hline Guarea macrophylla & 1625 & 1625 & & & & & & $\mathrm{CS}$ \\
\hline Guatteria australis & 50 & & & & 50 & 100 & -100 & $\mathrm{CL}$ \\
\hline Hieronyma alchorneoides & 250 & 200 & & & 50 & 20 & -20 & $\mathrm{CL}$ \\
\hline Hirtella hebeclada & 75 & 75 & & & & & & $\mathrm{CS}$ \\
\hline Hovenia dulcis & 25 & 25 & & & & & & $\mathrm{P}$ \\
\hline Inga lushnathiana & 50 & 50 & & & & & & $\mathrm{CS}$ \\
\hline Inga marginata & 425 & 375 & & & 50 & 12 & -12 & $\mathrm{CL}$ \\
\hline Inga sp. 1 & 2175 & 2225 & 300 & 14 & 250 & 11 & 2 & \\
\hline Inga striata $\mathrm{cf}$. & 25 & 25 & & & & & & $\mathrm{CS}$ \\
\hline Inga vera & 125 & 100 & & & 25 & 20 & -20 & $\mathrm{CS}$ \\
\hline Jacaranda puberula & 50 & 50 & & & & & & $\mathrm{CL}$ \\
\hline Lonchocarpus campestris & 775 & 1025 & 300 & 39 & 50 & 6 & 32 & $\mathrm{CL}$ \\
\hline Lonchocarpus sp. & 25 & 25 & & & & & & \\
\hline Luehea divaricata & 75 & 75 & & & & & & $\mathrm{CL}$ \\
\hline Machaerium stipitatum & 125 & 175 & 50 & 40 & & & 40 & $\mathrm{CL}$ \\
\hline Marlierea suaveolens & 25 & 25 & & & & & & $\mathrm{CS}$ \\
\hline Marlierea tomentosa & 1450 & 1650 & 200 & 14 & & & 14 & $\mathrm{CS}$ \\
\hline Matayba guianensis & 2375 & 2575 & 350 & 15 & 150 & 6 & 8 & $\mathrm{CS}$ \\
\hline Meliosma sellowii & 100 & 100 & & & & & & $\mathrm{CS}$ \\
\hline Miconia cabucu & 2475 & 2525 & 150 & 6 & 100 & 4 & 2 & $\mathrm{CL}$ \\
\hline Miconia cinnamomifolia & 825 & 625 & & & 200 & 24 & -24 & $\mathrm{P}$ \\
\hline Miconia sp. & 550 & 450 & & & 100 & 18 & -18 & \\
\hline Mollinedia clavigera & 25 & 25 & & & & & & $\mathrm{CS}$ \\
\hline Mollinedia schottiana & 1000 & 1000 & & & & & & $\mathrm{CS}$ \\
\hline Myrcia pubipetala & 14400 & 14500 & 550 & 4 & 450 & 3 & 1 & $\mathrm{CS}$ \\
\hline Myrcia rostrata & 550 & 600 & 50 & 9 & & & 9 & $\mathrm{CS}$ \\
\hline Myrsine coriacea & 275 & 275 & & & & & & $\mathrm{P}$ \\
\hline Nectandra rigida & 400 & 450 & 50 & 13 & & & 13 & $\mathrm{CS}$ \\
\hline
\end{tabular}




\begin{tabular}{|c|c|c|c|c|c|c|c|c|}
\hline \multirow{2}{*}{ Espécies } & \multicolumn{2}{|c|}{ Densidade } & \multicolumn{2}{|c|}{ Ingressos } & \multicolumn{2}{|c|}{ Mortalidade } & \multirow{2}{*}{$\begin{array}{c}\text { Mudança } \\
\%\end{array}$} & \multirow{2}{*}{$\begin{array}{c}\text { Grupo } \\
\text { ecológico }\end{array}$} \\
\hline & 2001 & 2003 & n.hat ${ }^{-1}$ & $\%$ & n.ha ${ }^{-1}$ & $\%$ & & \\
\hline NI5 & 100 & 200 & 100 & 100 & & & 100 & \\
\hline NI6 & 150 & 150 & & & & & & \\
\hline NI8 & 150 & 100 & & & 50 & 33 & -33 & \\
\hline Ocotea acyphilla & 50 & 50 & & & & & & $\mathrm{CS}$ \\
\hline Ocotea indecora & 25 & 25 & & & & & & $\mathrm{CS}$ \\
\hline Ocotea laxa & 200 & 200 & & & & & & $\mathrm{CS}$ \\
\hline Ocotea teleiandra & 225 & 225 & & & & & & $\mathrm{CL}$ \\
\hline Ormosia arborea & 100 & 100 & & & & & & $\mathrm{CL}$ \\
\hline Ouratea contarea & 1025 & 1125 & 150 & 15 & 50 & 5 & 10 & $\mathrm{CL}$ \\
\hline Pera glabrata & 2275 & 2400 & 150 & 7 & 25 & 1 & 5 & $\mathrm{CL}$ \\
\hline Piper cernuит & 50 & 50 & & & & & & $\mathrm{CS}$ \\
\hline Piper gaudichaudianum & 2825 & 2475 & 100 & 4 & 450 & 16 & -12 & $\mathrm{CS}$ \\
\hline Platymiscium floribundum & 50 & 50 & & & & & & $\mathrm{CL}$ \\
\hline Plinia sp. & 50 & 50 & & & & & & \\
\hline Posoqueria latifolia & 75 & 75 & & & & & & $\mathrm{CS}$ \\
\hline Protium kleinii & 100 & 100 & & & & & & $\mathrm{CS}$ \\
\hline Psidium catleyanum & 175 & 175 & & & & & & $\mathrm{CL}$ \\
\hline Psidium guajava & 25 & 25 & & & & & & $\mathrm{P}$ \\
\hline Psychotria kleinii & 75 & 75 & & & & & & $\mathrm{CS}$ \\
\hline Psychotria longipes & 18425 & 18050 & 700 & 4 & 1075 & 6 & -2 & $\mathrm{CS}$ \\
\hline Psychotria nuda & 5650 & 6875 & 1450 & 26 & 225 & 4 & 22 & $\mathrm{CS}$ \\
\hline Psychotria pubigera & 150 & 150 & & & & & & $\mathrm{CS}$ \\
\hline Psychotria sp. & 50 & 50 & & & & & & \\
\hline Psychotria suterella & 225 & 225 & & & & & & $\mathrm{CS}$ \\
\hline Psychotria tenerior & 100 & 100 & & & & & & $\mathrm{CS}$ \\
\hline Roupala brasiliensis & 25 & 25 & & & & & & $\mathrm{CL}$ \\
\hline Rudgea jasminoides & 25 & 25 & & & & & & $\mathrm{CS}$ \\
\hline Sapium glandulatum & 250 & 200 & 50 & 20 & 100 & 40 & -20 & $\mathrm{CL}$ \\
\hline Senna sp. & 125 & 125 & & & & & & \\
\hline Sloanea guianensis & 275 & 275 & & & & & & $\mathrm{CS}$ \\
\hline Sloanea sp. & 2175 & 1925 & & & 250 & 11 & -11 & \\
\hline Solanum swartzianum & 50 & 50 & & & & & & $\mathrm{P}$ \\
\hline Sorocea bonplandii & 725 & 775 & 100 & 14 & 50 & 7 & 7 & $\mathrm{CS}$ \\
\hline Strychnos brasiliensis & 25 & 25 & & & & & & $\mathrm{CS}$ \\
\hline Syzygium cumini & 25 & 25 & & & & & & $\mathrm{P}$ \\
\hline Talauma ovata & 50 & 50 & & & & & & $\mathrm{CL}$ \\
\hline Tocoyena sellowiana & 50 & 50 & & & & & & $\mathrm{CS}$ \\
\hline Trema micrantha & 400 & 450 & 50 & 13 & & & 13 & $\mathrm{P}$ \\
\hline Trichilia casarettoi & 175 & 175 & & & & & & $\mathrm{CS}$ \\
\hline Trichilia sp. & 25 & 25 & & & & & & \\
\hline Vantania compacta & 25 & 25 & & & & & & $\mathrm{CS}$ \\
\hline Vernonia puberula & 400 & 475 & 100 & 25 & 25 & 6 & 19 & $\mathrm{P}$ \\
\hline Vernonia sp. & 325 & 300 & & & 25 & 8 & -8 & $\mathrm{P}$ \\
\hline Virola bicuhyba & 100 & 75 & & & 25 & 25 & -25 & $\mathrm{CL}$ \\
\hline Xylopia brasiliensis & 225 & 225 & & & & & & $\mathrm{CL}$ \\
\hline Total & 73350 & 74700 & 5400 & & 4050 & & & \\
\hline
\end{tabular}

Em um estudo de taxas de crescimento e mortalidade em dipterocarpáceas na Malásia, Still (1996) obteve taxas de mortalidade de zero a $16 \%$ ao ano. Desse estudo resultou ainda que as taxas de 
mortalidade foram mais elevadas em indivíduos situados em sub-bosque mais denso e nas classes de alturas inferiores.

A

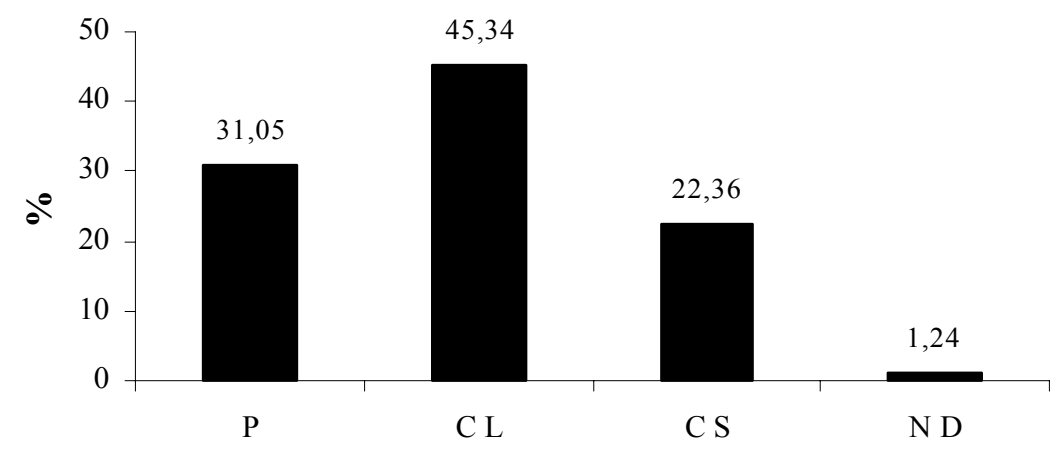

$\mathbf{B}$

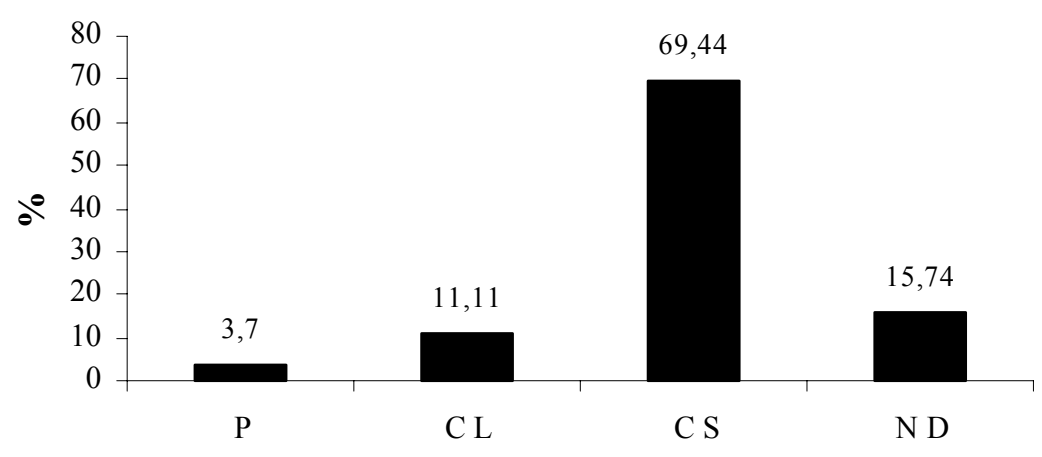

$\mathbf{C}$

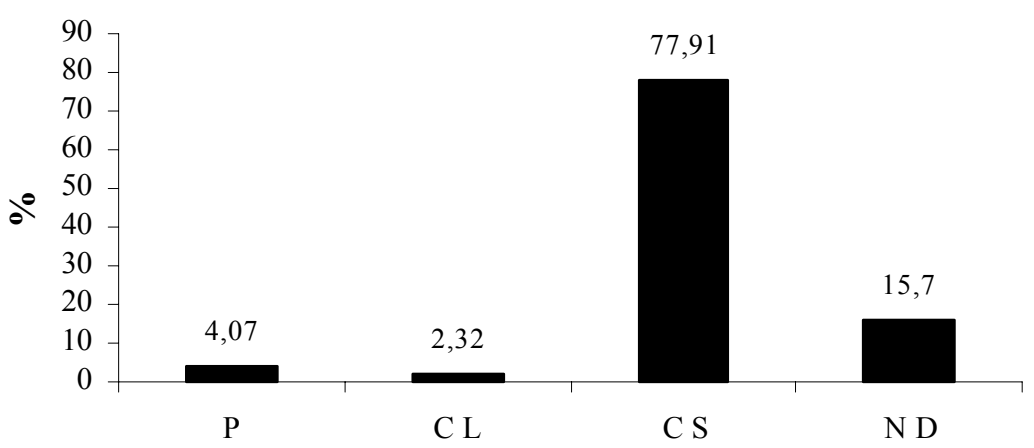

Figura 2. Ingressos nos grupos ecológicos e nos diferentes estágios sucessionais. (A = estágio inicial; B = estágio intermediário; $\mathrm{C}=$ estágio avançado; $\mathrm{P}=$ pioneiras; $\mathrm{CL}=$ clímax exigentes em luz; $\mathrm{CS}=$ clímax tolerantes à sombra; $\mathrm{ND}=$ não definidas).

Figure 2. Ingrow in the ecologic groups and in the different successional stages. $(\mathrm{A}=$ initial stage; $\mathrm{B}=$ intermediate stage; $\mathrm{C}=$ late stage; $\mathrm{P}=$ pioners; $\mathrm{CL}=$ climax light demanding; $\mathrm{CS}=$ climax shade tolerant; $\mathrm{ND}=$ no defined). 
A relação de 1,96 entre ingressos e mortalidade nas climácicas tolerantes à sombra indica que a floresta ainda encontra-se em processo de recomposição da estrutura, alterada em décadas passadas pela exploração seletiva. Estudos complementares deveriam ser realizados para indicar quais as mudanças que deverão ocorrer na dinâmica da floresta até ela atingir uma composição e estoqueamento considerados estáveis.

A
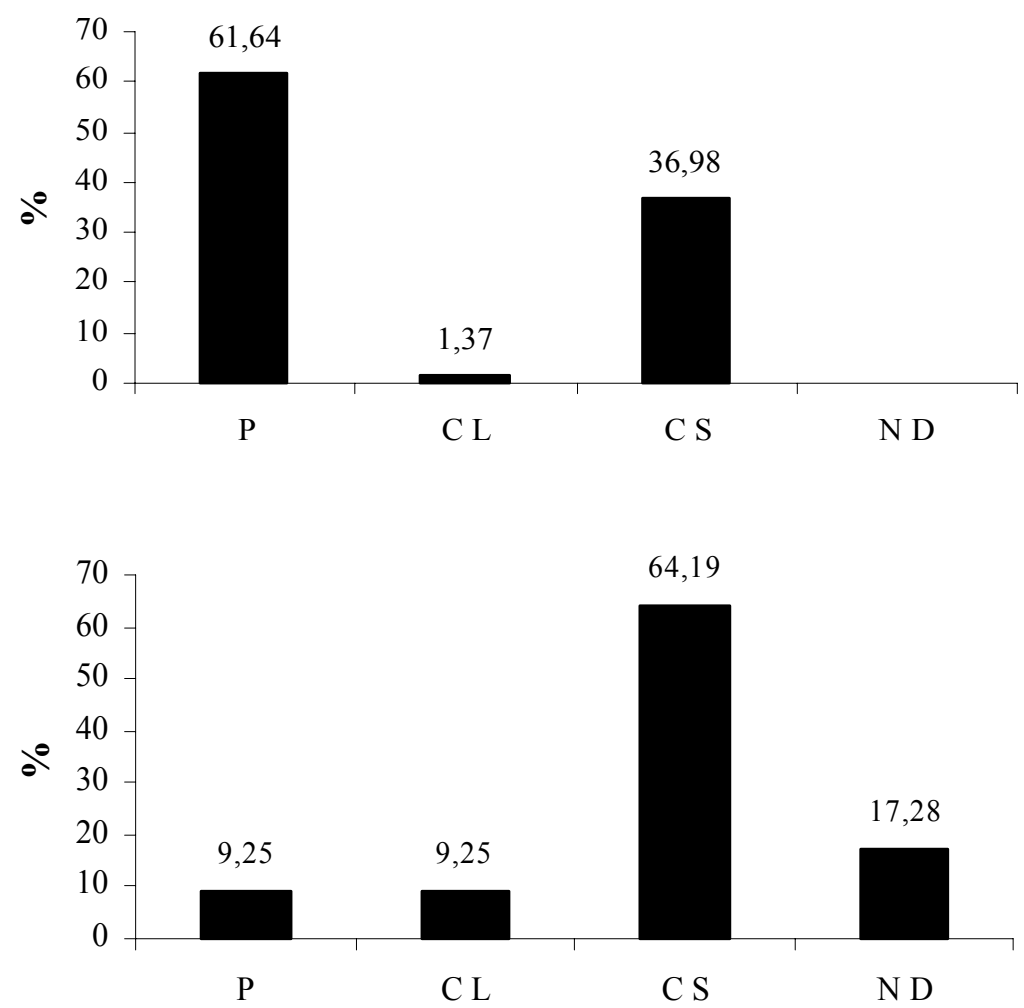

C

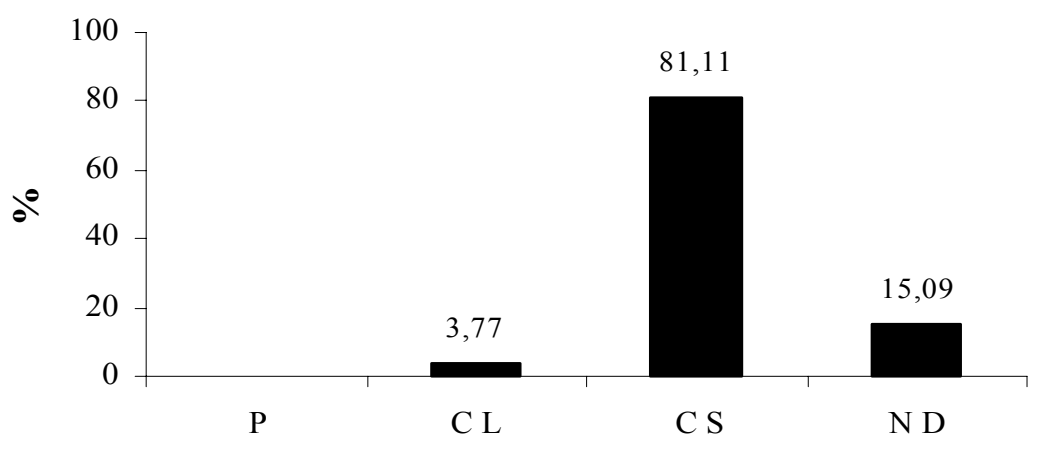

Figura 3. Mortalidade nos grupos ecológicos e nos diferentes estágios sucessionais. ( $\mathrm{A}=$ estágio inicial; $\mathrm{B}=$ estágio intermediário; $\mathrm{C}=$ estágio avançado; $\mathrm{P}=$ pioneiras; $\mathrm{CL}=$ clímax exigentes em luz; $\mathrm{CS}=$ clímax tolerantes à sombra; $\mathrm{ND}=$ não definidas).

Figure 3. Mortality in the ecologic groups and in the different successional stages. $(\mathrm{A}=$ initial stage; $\mathrm{B}=$ intermediate stage; $\mathrm{C}=$ late stage; $\mathrm{P}=$ pioners; $\mathrm{CL}=$ climax light demanding; $\mathrm{CS}=$ climax shade tolerant; $\mathrm{ND}=$ no defined). 
Tabela 3. Ingressos, mortalidade e porcentagem de mudança, por espécie, no estágio avançado.

Table 3. Ingrow, mortality and percent of changes, by species, in the changed late stage.

\begin{tabular}{|c|c|c|c|c|c|c|c|c|}
\hline \multirow{2}{*}{ Espécies } & \multicolumn{2}{|c|}{ Densidade } & \multicolumn{2}{|c|}{ Ingressos } & \multicolumn{2}{|c|}{ Mortalidade } & \multirow{2}{*}{$\begin{array}{c}\text { Mudança } \\
\%\end{array}$} & \multirow{2}{*}{$\begin{array}{c}\text { Grupo } \\
\text { ecológico }\end{array}$} \\
\hline & 2001 & 2003 & n.hat & $\%$ & n.ha ${ }^{-1}$ & $\%$ & & \\
\hline Aegiphylla sellowiana & 50 & 50 & & & & & & $\mathrm{CS}$ \\
\hline Annona cacans & 75 & 125 & 50 & 67 & & & 66,7 & $\mathrm{P}$ \\
\hline Bathysa meridionalis & 525 & 525 & & & & & & $\mathrm{CS}$ \\
\hline Bauhinia forficata & 50 & 100 & 50 & 100 & & & 100,0 & $\mathrm{P}$ \\
\hline Cabralea canjerana & 25 & 25 & & & & & & $\mathrm{CS}$ \\
\hline Casearia decandra & 50 & 50 & & & & & & $\mathrm{CL}$ \\
\hline Copaifera trapezifolia & 275 & 325 & 50 & 18 & & & 18,2 & $\mathrm{CS}$ \\
\hline Cyathea sp. & 325 & 325 & 50 & 15 & 50 & 15 & & $\mathrm{CS}$ \\
\hline Dalbergia brasiliensis & 75 & 100 & 25 & 33 & & & 33,3 & $\mathrm{CL}$ \\
\hline Dicksonia sellowiana & 50 & 50 & & & & & & $\mathrm{CS}$ \\
\hline Dodonea viscosa & 100 & 100 & & & & & & $\mathrm{P}$ \\
\hline Duguetia lanceolata & 25 & 25 & & & & & & $\mathrm{CS}$ \\
\hline Endlicheria paniculata & 175 & 175 & & & & & & $\mathrm{CS}$ \\
\hline Esenbeckia sp. & 475 & 600 & 125 & 26 & & & 26,3 & \\
\hline Euterpe edulis & 2550 & 3675 & 1525 & 60 & 400 & 16 & 44,1 & $\mathrm{CS}$ \\
\hline Faramea marginata & 50 & 50 & & & & & & $\mathrm{CS}$ \\
\hline Geonoma gamiova & 750 & 700 & & & 50 & 7 & $-6,7$ & $\mathrm{CS}$ \\
\hline Geonoma schottiana & 25 & 25 & & & & & & $\mathrm{CS}$ \\
\hline Guapira opposita & 375 & 375 & 50 & 13 & 50 & 13 & & $\mathrm{CS}$ \\
\hline Hieronyma alchorneoides & 100 & 125 & 25 & 25 & & & 25,0 & $\mathrm{CL}$ \\
\hline Hirtella hebeclada & 600 & 550 & & & 50 & 8 & $-8,3$ & $\mathrm{CS}$ \\
\hline Inga marginata & 400 & 475 & 75 & 19 & & & 18,8 & $\mathrm{P}$ \\
\hline Inga sessilis & 400 & 450 & 100 & 25 & 50 & 13 & 12,5 & $\mathrm{P}$ \\
\hline Inga sp. 1 & 350 & 550 & 250 & 71 & 50 & 14 & 57,1 & \\
\hline Inga vera & 50 & 50 & & & & & & $\mathrm{CS}$ \\
\hline Lonchocarpus campestris & 75 & 50 & & & 25 & 33 & $-33,3$ & $\mathrm{CS}$ \\
\hline Marlierea tomentosa & 850 & 875 & 25 & 3 & & & 2,9 & $\mathrm{CS}$ \\
\hline Matayba guianensis & 375 & 525 & 150 & 40 & & & 40,0 & $\mathrm{CS}$ \\
\hline Miconia cabucu & 150 & 175 & 25 & 17 & & & 16,7 & CL \\
\hline Miconia cinnamomifolia & 50 & 50 & & & & & & $\mathrm{P}$ \\
\hline Miconia sp. & 925 & 875 & & & 50 & 5 & $-5,4$ & \\
\hline Mollinedia schottiana & 150 & 200 & 200 & 133 & 150 & 100 & 33,3 & $\mathrm{CS}$ \\
\hline Myrcia pubipetala & 4350 & 4825 & 525 & 12 & 50 & 1 & 10,9 & $\mathrm{CS}$ \\
\hline Myrcia rostrata & 25 & 25 & & & & & & $\mathrm{CS}$ \\
\hline Myrsine coriacea & 50 & 50 & & & & & & $\mathrm{P}$ \\
\hline Nectandra rigida & 200 & 200 & 25 & 13 & 25 & 13 & & $\mathrm{CL}$ \\
\hline Ocotea catharinensis & 225 & 225 & & & & & & $\mathrm{CS}$ \\
\hline Ocotea odorifera & 100 & 100 & & & & & & $\mathrm{CS}$ \\
\hline Ocotea teleiandra & 125 & 125 & & & & & & $\mathrm{CL}$ \\
\hline Ormosia arborea & 50 & & & & 50 & 100 & $-100,0$ & CL \\
\hline Ouratea parviflora & 375 & 375 & & & & & & $\mathrm{CS}$ \\
\hline Pera glabrata & 25 & 25 & & & & & & $\mathrm{CL}$ \\
\hline Piper gaudichaudianum & 1175 & 1050 & & & 125 & 11 & $-10,6$ & $\mathrm{CS}$ \\
\hline Protium kleinii & 50 & 25 & 25 & 50 & 50 & 100 & $-50,0$ & $\mathrm{CS}$ \\
\hline Psychotria longipes & 3650 & 3450 & & & 200 & 5 & $-5,5$ & $\mathrm{CS}$ \\
\hline Psychotria nuda & 300 & 275 & & & 25 & 8 & $-8,3$ & $\mathrm{CS}$ \\
\hline Roupala brasiliensis & 75 & 75 & & & & & & $\mathrm{CL}$ \\
\hline Senna sp. & 25 & 25 & & & & & & \\
\hline Sloanea guianensis & 500 & 450 & & & 50 & 10 & $-10,0$ & $\mathrm{CS}$ \\
\hline Solanum swartzianum & & 25 & 25 & & & & & $\mathrm{P}$ \\
\hline
\end{tabular}




\begin{tabular}{|c|c|c|c|c|c|c|c|c|}
\hline \multirow{2}{*}{ Espécies } & \multicolumn{2}{|c|}{ Densidade } & \multicolumn{2}{|c|}{ Ingressos } & \multicolumn{2}{|c|}{ Mortalidade } & \multirow{2}{*}{$\begin{array}{c}\text { Mudança } \\
\%\end{array}$} & \multirow{2}{*}{$\begin{array}{c}\text { Grupo } \\
\text { ecológico }\end{array}$} \\
\hline & 2001 & 2003 & n.ha ${ }^{-1}$ & $\%$ & n.ha ${ }^{-1}$ & $\%$ & & \\
\hline Sorocea bonplandii & 25 & 50 & 25 & 100 & & & 100,0 & $\mathrm{CS}$ \\
\hline Trema micrantha & 100 & 100 & & & & & & $\mathrm{P}$ \\
\hline Trichilia sp. & 850 & 875 & 75 & 9 & 50 & 6 & 2,9 & \\
\hline Vantania compacta & 400 & 375 & 25 & 6 & 50 & 13 & $-6,3$ & $\mathrm{CS}$ \\
\hline Vernonia puberula & 50 & 75 & 25 & 50 & & & 50,0 & $\mathrm{P}$ \\
\hline Virola bicuhyba & 300 & 275 & & & 25 & 8 & $-8,3$ & $\mathrm{CS}$ \\
\hline Xylopia brasiliensis & 25 & & & & 25 & 100 & $-100,0$ & $\mathrm{CL}$ \\
\hline Total & 23650 & 25525 & 3525 & & 1650 & & 7,9 & \\
\hline
\end{tabular}

\section{Síntese da dinâmica nos estágios sucessionais}

Com base nos resultados, é possível inferir que as espécies apresentam padrões de comportamento, quanto às taxas de ingresso e de mortalidade, de acordo com o grupo ecológico ao qual pertencem e à fase sucessional onde se encontram.

Enquanto as taxas de ingresso das espécies pioneiras e climácicas exigentes em luz diminuem do estágio inicial para avançado, as climácicas tolerantes à sombra apresentam tendência de incremento dos ingressos. Comportamento idêntico foi obtido quanto à mortalidade nos grupos ecológicos e nos diferentes estágios (Figuras 1 e 2). A mortalidade no estágio intermediário e no avançado, especialmente das espécies climácicas tolerantes à sombra, é amparada pelas considerações de Richard (1952), GomezPompa e Wiechers (1976) e Brokaw (1985a), de que essas espécies são apenas parcialmente tolerantes à sombra, pois dependem do aumento nos níveis de luz para chegar à maturidade. Carvalho (1997) também compartilhou desse princípio ao citar a ocorrência de taxas de mortalidade mais elevadas em espécies de sub-bosque, quando comparadas com espécies emergentes.

Os padrões de ingresso e mortalidade apresentados pelas espécies estão relacionados às estratégias de estabelecimento dos respectivos grupos ecológicos. A estratégia de formação de banco de plântulas, característica das clímax tolerantes à sombra, é mais importante em comunidades com pequenas clareiras ou com limitação de luminosidade (BAZZAZ, 1991).

A densidade da regeneração natural em 2001 mostrou uma tendência de formação de banco de plântulas, tanto no estágio intermediário quanto no avançado, e reforça as considerações de Bazzaz (1984) sobre as características da germinação de sementes e estabelecimento de plântulas.

Observa-se, na tabela 4, que as pioneiras, em termos de ingresso, apresentaram comportamento semelhante no estágio intermediário e no avançado, cujas condições ambientais são caracterizadas pela limitação da luminosidade nos estratos inferiores. Por outro lado, os ingressos de espécies clímax tolerantes à sombra nessas duas fases foram elevados e estatisticamente semelhantes.

Tabela 4. Ingressos e mortalidade, por hectare, por grupo ecológico, nos três estágios sucessionais, entre 2001 e 2003.

Table 4. Ingrow and mortality, per hectare, per ecologic group, in the tree successional stages, between 2001 and 2003.

\begin{tabular}{lccrcrr}
\hline \multirow{2}{*}{ Grupo Ecológico } & \multicolumn{2}{c}{ Estágio Inicial } & \multicolumn{2}{c}{ Estágio Intermediário } & \multicolumn{2}{c}{ Estágio Avançado } \\
\cline { 2 - 7 } & Ingressos & Mortalidade & Ingressos & Mortalidade & Ingressos & Mortalidade \\
\hline $\mathrm{P}$ & $1250 \mathrm{~A} \mathrm{a}$ & $1125 \mathrm{~A} \mathrm{a}$ & $200 \mathrm{~B} \mathrm{a}$ & $275 \mathrm{~B} \mathrm{a}$ & $325 \mathrm{~B} \mathrm{a}$ & $50 \mathrm{C} \mathrm{a}$ \\
$\mathrm{CL}$ & $1975 \mathrm{~A} \mathrm{a}$ & $25 \mathrm{~A} \mathrm{~b}$ & $900 \mathrm{~B} \mathrm{~b}$ & $525 \mathrm{~B} \mathrm{~b}$ & $100 \mathrm{C} \mathrm{b}$ & $100 \mathrm{~A} \mathrm{~b}$ \\
$\mathrm{CS}$ & $625 \mathrm{~A} \mathrm{~b}$ & $675 \mathrm{~A} \mathrm{c}$ & $3850 \mathrm{~B} \mathrm{c}$ & $2600 \mathrm{~B} \mathrm{~b}$ & $2650 \mathrm{~B} \mathrm{c}$ & $1350 \mathrm{C} \mathrm{c}$ \\
$\mathrm{ND}$ & $125 \mathrm{~A} \mathrm{c}$ & & $450 \mathrm{~A} \mathrm{~b}$ & $650 \mathrm{~A} \mathrm{a}$ & $450 \mathrm{~A} \mathrm{~d}$ & $150 \mathrm{~A} \mathrm{a}$ \\
\hline Total & 3975 & 1825 & 5400 & 4050 & 3525 & 1650 \\
\hline
\end{tabular}

$\mathrm{P}=$ pioneiras $; \mathrm{CL}=$ clímax exigentes em luz; $\mathrm{CS}=$ clímax tolerantes à sombra; $\mathrm{ND}=$ não definidas.

Médias seguidas da mesma letra, maiúscula nas linhas e minúscula nas colunas, não diferem entre si pelo teste $\mathrm{F}(\mathrm{P}>0,05)$. 
O ingresso mais elevado de espécies clímax tolerantes à sombra no estágio intermediário em relação ao avançado pode ser resultado de diferenças de pressão de competição, causadas por diversos fatores ambientais, intrínsecos e antrópicos, conforme mencionaram Whitmore $(1978,1984)$ e Silva (1989).

No estudo da dinâmica de regeneração natural após a extração seletiva na Floresta de Tapajós, Silva (1989) obteve um decréscimo de $34 \%$ no total de plântulas, em um período de 6 anos. Considerou ainda que essa flutuação na população de plântulas pode ser resultado de diferentes freqüências de frutificação, manifestadas pelas diferentes espécies.

Embora o período entre as avaliações neste trabalho tenha sido bastante curto, foi possível detectar nos estágios analisados padrões e tendências observados por outros autores em florestas tropicais (BROKAW, 1985b; BAZZAZ, 1991): as espécies mais importantes nos estágios iniciais apresentam instalação e crescimento rápidos, mas em espaço de tempo limitado, enquanto que as espécies dominantes em estágios avançados apresentam crescimento mais lento e contínuo.

Still (1996) também considerou que nas classes de alturas superiores, nas quais o crescimento é lento e o intervalo de tempo necessário para mudanças de classes é maior, o risco de mortalidade é elevado, o que explica, de certa forma, a elevada taxa de mortalidade de espécies clímax tolerantes à sombra no estágio avançado.

Deve-se considerar ainda que os resultados podem ter sido influenciados por fatores relacionados à amostragem. O período de levantamento em campo, nas duas ocasiões, foi de dezembro a maio, não abrangendo, portanto, toda a estação de crescimento.

A comparação dos resultados com aqueles obtidos em outros trabalhos também deve ser vista com ressalvas, pois, em geral, são usadas metodologias ou intensidades de amostragem diferentes.

\section{CONCLUSÕES}

- Os ingressos no estágio inicial foram mais expressivos em espécies climácicas exigentes em luz, principalmente Miconia cabucu, Hieronyma alchorneoides, Miconia cinnamomifolia. Nos estágios intermediário e avançado, as espécies clímax tolerantes à sombra apresentaram os maiores ingressos.

- A mortalidade no estágio inicial foi mais intensa nas espécies pioneiras, enquanto que nos estágios intermediário e avançado foi maior em espécies clímax tolerantes à sombra.

- $\quad \mathrm{Na}$ regeneração natural do estágio inicial, as espécies pioneiras estão sendo gradativamente substituídas por espécies clímax exigentes em luz, e em menor escala pelas clímax tolerantes à sombra.

- No estágio intermediário, as espécies pioneiras e clímax exigentes em luz, atualmente mais importantes na estrutura do estrato superior, estão sendo substituídas por espécies clímax tolerantes à sombra.

\section{REFERÊNCIAS}

AUGSPURGER, C. K. Seedling survival of tropical tree species: interactions of dispersal distance, light gaps and pathogens. Ecology, v. 65, p. 1705-1712, 1984.

BAZZAZ, F. A. Dynamics of wet tropical forest and their species strategies. In: Medina, E., Mooney, H. A., Vasquez-Yanes, C. (eds.) Physiological ecology of plants of the wet tropics. The Hague, 1984. p. 233-243.

BAZZAZ, F. A. Regeneration of tropical forests: physiological responses of pioneer and secondary species. In: Gómez-Pompa, A.; Whitmore, T. C.; Hadley, M. Rain forest regeneration and management. Paris: Man and The Biosphere Series. 1991. vol. 6. p. 91-114.

BROKAW, N. V. L. Gap-phase regeneration in a tropical forest. Ecology. N. 66, p. 682-687, 1985 b.

CARVALHO, J. O P. Dinâmica de florestas naturais e sua Implicação para o manejo florestal. In: Curso de Manejo Florestal Sustentável. Colombo: EMBRAPA, 1997. 250 p.

DAUBENMIRE, R. Plant Communities. New York: Harper \& Row Publishers, 1968. 300 p. 
DENSLOW, J. S. Tropical rainforest gaps and tree species diversity. Annual Review of Ecology and Systematics v. 18, p. 431-451, 1987.

FINOL, U. H. Nuevos parâmetros a considerarse em el analisis estructural de lãs selvas virgenes tropicales. Rev. For. Venezoelana. v. 14, n. 21, p. 29-49, 1971.

GOMEZ-POMPA, A; WIECHERS, B. L. Regeneración de los ecosistemas tropicales y subtropicales. In: GOMEZ-POMPA, A; VÁSQUES-YANES, C.; AMO RODRIGUES, S.; CERVERA, A. B. Investigaciones sobre la regeneración de las selvas altas en Veracruz, México. México: Continental, 1976. p 11-30.

GRAU, H. R. Regeneration patterns of Cedrela lilloi (Meliaceae) in northwestern Argentina subtropical montane forests. Journal of Tropical Ecology. v. 16, p. 227-242, 2000.

GRUBB, P. J. The maintenance of species richness in plant communities. The importance of the regeneration niche. Biological Review, v. 52, p. 45-107, 1977.

HARPER, J. L. Population biology of plants. London: Academic Press, 1977.

PICKETT, S. T. A.; OSTFELD, R. S. The shifting paradigm in ecology. In: KNIGHT, R.L.; BATES, S.F. (Ed.) A new century for natural resources management. Washington: Islands Press, 1995. p. 261-278.

POPMA, J.; BONGERS, F. Acclimation of seedlings of three Mexican tropical rain forest tree species to a change in light availability. Journal of Tropical Ecology, n. 7, p. 85-97, 1991.

PRIMACK, R. B.; LEE, H. S. Populations dynamics of pioneer (Macaranga) trees and understorey (Mallotus) trees (Euphorbiaceae) in primary and selectively logged Bornean rain forests. Journal of Tropical Ecology, n. 7, p. 439-458, 1991.

RICHARDS, P. W. The tropical rain forest. Cambridge: Cambridge Univ. Press, 1952. 450 p.

RODRIGUES, R. R. Colonização e enriquecimento de um fragmento florestal urbano após a ocorrência de fogo. Fazenda Santa Elisa, Campinas, SP: Avaliação temporal da regeneração natural (66 meses) e do crescimento (51 meses) de 30 espécies florestais plantadas em consórcios sucessionais. Piracicaba: 1999. Tese (Livre Docência) - Escola Superior de Agricultura Luiz de Queiroz, Universidade de São Paulo.

ROLLET, B. Arquitetura e crescimento das florestas tropicais. Belém: SUDAN, 1978. 22 P.

SILVA, J. N. M. The behaviour of the Tropical Rain Forest of the Brazilian Amazon after logging. Oxford: 1989, $303 \mathrm{f}$. Thesis (Doctor of Philosophy) University of Oxford.

SORREANO, M. C. M. Avaliação de aspectos da dinâmica de florestas restauradas com diferentes idades. Piracicaba: 2002. Dissertação (Mestrado em Engenharia Florestal) Escola Superior de Agricultura Luiz de Queiroz.

STERCK, F. J.; CLARK, D. B.; CLARK, D. A.; BONGERS, F. Light fluctuations, crown traits, and response delays for tree saplings in a Costa Rican lowland rain forest. Journal of Tropical Ecology. v. 15, p. 83-95, 1999

STILL, M. J. Rates of martality and growth in three groups of dipterocarp seedlings in Sabah, Malaysia. In: The Ecology of Tropical Forest Seedlings. Paris: Parthenon Publishing, 1996. p. 315-331.

TORIOLA, D.; CHAREYRE, P.; BUTTLER, A. Distribution of a primary forest plant species in a $18^{\circ}$ year old secondary forest in French Guiana. Journal of Tropical Ecology, v.14, n. 3, p. 323-340, 1998.

VEBLEN, T. T. Regeneration dynamics. In: Plant Succession. Theory and prediction. London: Chapman and Hall, 1992. p. 153-187.

VOLPATO, M. M. L. Regeneração natural em uma floresta secundária no domínio de mata atlântica: uma análise fitossociológica. Viçosa: 1994. 123 p. Dissertação (Mestrado em Engenharia Florestal) - Universidade Federal de Viçosa. 
WHITMORE, T. C. Gaps in the forest canopy. In: Tomlinson, P. B., e Zimmerman, M. H. (eds.) Tropical trees as living systems. New York: Cambridge University Press, 1978 p. 639-655.

WHITMORE, T. C. Tropical rain forest of the Far East. $2^{\mathrm{a}}$ ed. Oxford: Clarendon Press, 1984. 352 p.

WHITMORE, T.C. An introduction to tropical rain forest. $2^{\mathrm{a}}$ ed. Oxford: Clarendon Press, 1990. 